\title{
Jóvenes y consumo de noticias a través de Internet y los medios sociales
}

\author{
Beatriz CATAlina-García \\ Universidad Rey Juan Carlos \\ beatriz.catalina@urjc.es \\ Antonio GARCíA JimÉNEZ \\ Universidad Rey Juan Carlos \\ antonio.garcia@urjc.es \\ Manuel Montes Vozmediano \\ Universidad Rey Juan Carlos \\ manueljavier.montes@urjc.es
}

Recibido: 17 de octubre de 2015

Aceptado: 4 de diciembre de 2015

\section{Resumen}

El objetivo de este trabajo es analizar los hábitos de los jóvenes en relación al consumo de las noticias en Internet en un escenario donde los medios sociales tienen una gran presencia. Una encuesta realizada entre universitarios españoles permite estudiar su comportamiento a partir, por un lado, de la intensidad de acceso y, por otro, del género, tomando en consideración la frecuencia en la consulta de noticias, el medio seleccionado, la motivación para informarse, el contraste de noticias, así como la percepción sobre la imparcialidad y la credibilidad. La investigación permite constatar la creciente relevancia de los medios sociales como fuente para el acceso a la información de actualidad.

Palabras clave: noticias; juventud; Internet; medios de comunicación; medios sociales.

\section{Youths and consumption of news via Internet and social media}

\begin{abstract}
The aim of this paper is to analyze the habits of young people in relation to consumption of news on the Internet, in a scenario where social media has a strong presence. A survey of Spanish university students focused on, on the one hand, on the intensity of access and, secondly, on gender, is carried out in relation to the frequency of news access, the specialization of journalistic information, the motivation to read news, the news verifying and the perception about impartiality and credibility. This exploratory research has revealed the growing importance of social media as a source for access to news.
\end{abstract}

Keywords: news; youth; Internet; mass media; social media.

\section{Referencia normalizada}

Catalina García, B.; García Jiménez, A.; Montes Vozmediano, M. (2015). Jóvenes y consumo de noticias a través de Internet y los medios sociales. Historia y Comunicación Social. Vol 20, número 2, páginas 601-619. 
Sumario: 1. Introducción. 2. Estado de la cuestión. 2.1. Búsqueda y credibilidad de la información en Internet. 2.2. Consumo de noticias y jóvenes. 2.3. Noticias y medios sociales. 3. Objetivos e hipótesis. 4. Metodología. 5. Resultados. 5.1. Preferencia para la consulta. 5.2. Frecuencia de acceso. 5.3. Especialización de la información. 5.4. Motivos para consultar las noticias. 5.5. Verificación de la información de actualidad. 5.6. Distribución de noticias. 5.7. Percepción del grado de imparcialidad y credibilidad en los distintos medios. 6. Conclusiones y discusión. 7. Referencias bibliográficas.

\section{Introducción}

La constatación del vertiginoso cambio en el comportamiento de los jóvenes en su acercamiento al dominio mediático es el motor de este trabajo. En los últimos años, se ha detectado el creciente acceso a los medios de comunicación en línea y el fuerte impacto de los medios sociales.

Son muchos los interrogantes teóricos y prácticos que se deben despejar. Algunos de ellos se abordan en este texto, que se pregunta por las conductas y hábitos de los estudiantes universitarios para informarse sobre los hechos de actualidad. Interesa conocer las vías que utilizan para este fin y, en particular, analizar si el uso habitual que realizan los jóvenes de los medios sociales incluye este cometido, ya sea para complementar o para reemplazar la consulta de noticias a través de los medios de comunicación tradicionales (televisión, prensa escrita y radio), o de sus versiones online.-

\section{Estado de la cuestión}

\subsection{Búsqueda y credibilidad de la información en Internet}

La primera cuestión analizada es la relativa al acceso, uso y percepción sobre la información en Internet y en los medios sociales. Estudios como el de Kim et al. (2013) confirman la relevancia de la búsqueda de información a través de las redes sociales por parte de los jóvenes universitarios. En realidad, se convierte en una actividad que viene determinada tanto por rasgos de personalidad como por las disciplinas estudiadas, el género o el tipo de acción digital de que se trate.

Por su parte, García et al. (2014), al analizar en los universitarios españoles la "dieta de servicios digitales", definidos estos como herramientas y espacios de la web social que ofrecen información y recursos con acceso libre, observan que cuanto mayor y variada sea esta dieta, aumenta la confianza y la percepción positiva sobre la utilidad de la información accesible en Internet. De igual forma, sostienen que son los denominados usuarios intensivos los que hacen un mayor uso de estos servicios digi- 
tales y depositan un mayor grado de confianza en la información digital. Asimismo, la dieta informacional de los jóvenes universitarios se caracteriza por el escaso uso de los medios que tienen a su disposición. O dicho de otra manera, hacen un uso intensivo de un conjunto limitado de servicios y fuentes. Esta afirmación se encuentra en la línea de lo que señalan Hasebrink y Domeyer (2012), pero choca con la propia idea de "nativos digitales", dados sus supuestos conocimientos tecnológicos.

Otro aspecto relevante es la relación existente entre la orientación marcada por el usuario y el aumento de la credibilidad. De hecho, los individuos marcan unos objetivos concretos a la hora de buscar información y, como apunta el trabajo de Fogg (2002), a mayor necesidad y urgencia por acceder a la información, se eleva la posibilidad de considerar creíbles los contenidos que se encuentran en la web.

Por otra parte, y todavía fuera de lo que se circunscribe a la información periodística, Sánchez-Navarro y Aranda (2011) sostienen que son tres los criterios que emplean los jóvenes para evaluar la veracidad/utilidad o confianza en la información: por un lado, lo que podría entrar en la esfera de los conocimientos previos, alejados del aprendizaje formal. El siguiente criterio es el denominado prestigio de la fuente. Curiosamente, esta afirmación podría estar reñida con una cultura más orientada a la versión wiki y compartida del conocimiento. El tercer criterio está vinculado a la idea de "reiteración", es decir, la posibilidad de acceder a la misma información a través de diferentes fuentes. A esto habría que añadir la confianza que procede de las redes de iguales, amigos y conocidos (Melton et al., 2012).

Por último, se constata que los medios sociales, considerados en un sentido amplio, son ya una fuente popular de acceso a la información. De hecho, Wikipedia obtiene un $98.6 \%$ de uso, Facebook un $95.7 \%$, los blogs un $32 \%$ y Twitter un $25 \%$. Los jóvenes emplean las redes sociales en primer lugar para mantenerse en contacto con otros $\mathrm{y}$, a continuación, para obtener actualizaciones, noticias y otras opiniones. Resulta relevante el hecho de que Twitter se utilice menos como fuente de información, si bien es evidente su creciente papel como espacio de localización de información, dada su fuerza para diseminar la información de actualidad (Kim et al., 2014)

\subsection{Consumo de noticias y jóvenes}

Centrados ya en el consumo de noticias, Condeza, Bachmann y Mujica (2014) detectan tres motivaciones fundamentales: la vigilancia o monitoreo de la actualidad, el entretenimiento y lo que se puede denominar como utilidad social. Los adolescentes aseguran que es importante estar en contacto con la actualidad. Con esta afirmación se manifestó algo de acuerdo el 54,6\%, y un 29,3\% muy de acuerdo. A su vez, entienden que estar en contacto con las noticias obedece a su interés por los problemas de las personas (casi un 50\% estuvo algo de acuerdo, y un 32\% muy de acuerdo). Este planteamiento se mantiene en el tiempo, al igual que sus preferencias temáticas (Túñez, 2009).

Entre otros autores, Casero Ripollés (2012) confirma el progresivo consumo informativo a través de los nuevos espacios, fundamentalmente los medios sociales, mien- 
tras baja en los medios tradicionales y digitales; que los jóvenes estén interesados en las noticias se asocia en gran medida a su valor cívico. Además, acuden a fuentes muy variadas y de modo fragmentado, haciéndolo compatible con otras actividades digitales. Se trata de un consumo que se incrementa con la edad en términos de interés, frecuencia de consulta y variedad de las fuentes. Destaca también cierta brecha de género: los varones presentan un mayor índice lectura, mientras las mujeres inciden más en el valor social y ciudadano de las noticias.

Es creciente en este sector de la población la comprensión de la noticia más como un modo de servicio público que como producto. En este sentido, Costera (2007) apunta que los jóvenes creen que las noticias deben estar disponibles para los ciudadanos, y por ende, tender a la gratuidad, en un medio dominado por la inmediatez. Pero al mismo tiempo que se produce el aumento al respecto de su consideración cívica, aumenta la percepción de que las noticias son un producto sin gran valor material. Quizá esto se puede relacionar con la relevancia que se da a la gratuidad, hecho vinculado y con fuerte arraigo entre los más jóvenes.

\subsection{Noticias y medios sociales}

Existen indicios suficientes para pensar en la transformación durante los últimos años, que ha experimentado la relación entre jóvenes, información de actualidad y medios sociales. Algunos primeros estudios indicaban (Patterson, 2007; Qay Yum et al., 2010) que el consumo informativo mediante búsqueda directa no se constituía como una actividad preferente de los jóvenes. Más bien aparece cuando el joven advierte que algo le resulta interesante en el ciberespacio.

Sin embargo, Condeza, Bachmann y Mujica (2014) han comprobado que los medios sociales, junto a la televisión, representan para los adolescentes un espacio muy importante para informarse. Por ejemplo, gracias al artículo de Hermida et al. (2012), se puede ver que el $40 \%$ de los usuarios de las redes sociales recibe noticias mediante plataformas como Facebook, mientras que otro 20\% las obtiene de las empresas de comunicación y de periodistas de los que son seguidores. De igual modo, estos usuarios valoran los medios sociales porque le ayudan a mantenerse en contacto con la realidad con una amplia gama de noticias. En definitiva, es clara la tendencia al empleo de los medios sociales como herramienta informativa, lo que podría sugerir cambios, a diferentes niveles, tanto en la producción de información como en la recepción de la misma.

Esta percepción también es confirmada por Gangadharbatla et al. (2014). Los jóvenes ya no plantean una sola forma de acceso a las noticias. De hecho, tienen la sensación de que los medios sociales les otorgan mayor control sobre la información que sobre la recibida por parte de los medios tradicionales. Además, entienden que proporcionan mayor flexibilidad a la hora de recopilar datos, aunque en muchos casos conceden más credibilidad a los medios tradicionales. Esta aparente desconexión entre una opinión y otra demuestra la complejidad del fenómeno aquí estudiado. Una posible explicación se deriva de que los jóvenes pueden tener el primer contacto con 
un hecho en los medios sociales, pero después acuden al medio tradicional para tareas de confirmación y verificación. Otra explicación está relacionada con la tecnología "self-efficacy". Los individuos optan por una herramienta que puede ser útil para determinados usos, y esto es lo que ocurriría con el acceso a las noticias, anteponiendo esta variante a otros criterios.

En el estudio García Avilés et al. (2014) se observa que se da un respetable nivel de credibilidad a las noticias canalizadas por los medios sociales, en gran medida por la posibilidad de participar, así como por la visibilidad e impacto de la actividad los periodistas, aunque siga siendo decisiva la imagen del medio de comunicación que haya publicado una noticia. Este hallazgo, no obstante, no se corrobora en todos los casos. Johnson y Kaye (2014), en el marco de la información y comunicación política, detectan que los medios sociales son considerados menos creíbles que el resto de fuentes online y tradicionales.

Por otra parte, Turcotte et al. (2015) encuentran clave el papel de recomendaciones de los medios sociales, fundamentalmente en Facebook. Esta opción en realidad mejora los niveles de confianza hacia los medios de comunicación, y al mismo tiempo consigue que los individuos deseen seguir más noticias. Este fenómeno aumenta si se percibe por parte de los jóvenes la presencia de líderes de opinión.

Finalmente, Gil de Zúñiga, Jung y Valenzuela (2012) profundizan en cómo el uso de los medios digitales para objetivos informacionales también contribuye a fomentar los procesos democráticos y la creación de capital social. La búsqueda de información de actualidad a través de los medios sociales se configura, entre los estudiantes, como un indicador relevante y significativo del capital social de las personas y de los comportamientos participativos de naturaleza política, tanto online como offline. Y esto se produce a pesar de que la mayor parte del intercambio de información en los medios sociales no esté vinculado a temas públicos o noticias, sino por ejemplo al entretenimiento.

\section{Objetivos e hipótesis}

El objetivo de este trabajo es analizar las conductas y hábitos de los estudiantes universitarios en relación al consumo de las noticias. La clave se sitúa en si los medios sociales, al margen de otros usos que los jóvenes hagan de ellos, se han convertido en fuente para el acceso a noticias, en clara competencia con los tradicionales medios de comunicación. Las dos variables que delimitan este estudio son: por un lado, la frecuencia e intensidad de acceso a Internet y, por otro lado, el género. Las hipótesis de trabajo desde las que se parte son:

Hipótesis 1. Los jóvenes recurren preferentemente a los medios sociales para el consumo de información de actualidad, independientemente de otras tareas que pudieran realizar en ellas. 
Hipótesis 2. Una mayor intensidad en el acceso digital modifica el consumo de noticias entre los jóvenes, pero también esta intensidad les influye en otros aspectos y conductas, a saber: en la selección del medio de comunicación, en las preferencias que los jóvenes tienen sobre el tipo de información, las motivaciones que les incita a informarse, en su predisposición a contrastar las informaciones y en las formas que utilizan para distribuirlas, así como en su percepción sobre la imparcialidad y sobre la credibilidad que les inspiran los diferentes medios y vías de comunicación.

Hipótesis 3. La variable de género no es determinante en el consumo de noticias en Internet.

\section{Metodología}

El estudio se ha realizado sobre la base de una encuesta de carácter exploratorio distribuida y auto-administrada entre alumnos que cursaban las siguientes asignaturas, cursos y grados en la Universidad Rey Juan Carlos (Madrid):

- Investigación de Audiencias y Planificación de Medios. Tercer curso del grado de Publicidad y Relaciones Públicas.

- Teoría de la Información. Primer curso del doble grado de Periodismo y Ciencias Políticas.

- Teoría de la Información. Primer curso del grado de Comunicación Audiovisual.

- Fotografía Digital. Tercer curso del grado de Bellas Artes. Se aplica un método de muestreo no probabilístico.

En total se han extraído los datos de 297 encuestas cumplimentadas. El cuestionario fue distribuido en las aulas durante el mes de mayo de 2015. Constaba de 20 preguntas, 7 de ellas con carácter de respuesta múltiple y 2 sirvieron de filtro. El tiempo requerido para completarlo oscilaba entre los 5 y los 10 minutos.

Las edades de los participantes en este estudio oscilan entre los 18 y los 30 años, con una media resultante de 20,94 años y una moda de 21 . La muestra no es representativa del colectivo juvenil español, hecho que constituye la primera limitación de este análisis, aunque sí ofrece resultados productivos de cara a futuras investigaciones. Una segunda limitación se deriva de las condiciones del universo de estudio cuyo rasgo fundamental es la desigualdad en las proporciones de la muestra: el índice de mujeres $(68,4 \%)$ es muy superior al de los hombres encuestados $(31.3 \%)$.

Un $2 \%$ de los encuestados afirmó que no consulta nunca las noticias. En este sentido, la mayoría $(83,3 \%)$ se conforma con la información que les proporcionan otras personas, un $16 \%$ reconoce que no está interesado, $\mathrm{y}$, en proporciones iguales $(33,3 \%)$, alegan que ningún medio les inspira confianza, que no es una prioridad para ellos o que no tienen tiempo. A todos ellos se les invitó a no continuar con el cuestionario. Una vez explicado el propósito de la investigación, se sugirió a los estu- 
diantes que lo cumplimentaran atendiendo al anonimato de los datos que expusieran e instándoles, por tanto, a que contestaran con el mayor rigor y sinceridad posible.

Para la consecución del análisis se han tenido en cuenta los diferentes aspectos que tienen que ver con la consulta y percepción de la actualidad por los jóvenes. En función del género y la intensidad o frecuencia de navegación diaria por Internet, se han respondido a las siguientes cuestiones:

- Preferencia por los medios de comunicación online o tradicionales. Se planteó por un lado, el orden de predilección de los distintos medios tradicionales para la consulta de noticias; y por otro, en formato de respuesta múltiple, qué modos empleaban para informarse: en primer lugar, las versiones online de los medios tradicionales; los medios sociales (blogs, redes sociales) como segunda opción; en tercer lugar las aplicaciones de mensajería como What$s A p p$, y finalmente, los buscadores y otros recursos. También se incluyó la respuesta de "ninguno" para aquellos encuestados que no recurrieran a Internet para la consulta de información.-

- Frecuencia de consulta de noticias. Los encuestados optaban por cuatro opciones que discurrían entre "no leer las noticias todos los días" y "realizar tres o más consultas diarias". Se incluía también la alternativa de "nunca" que servía como respuesta filtro para no continuar con el cuestionario.

- Tipos de información que más consultan. Para la construcción de esta pregunta se utilizaron las habituales secciones y temáticas en las que tradicionalmente se han dividido los contenidos de los medios de comunicación:

- Política. Incluyendo los ámbitos nacional e internacional.

- Economía.

- Deportes.

- Sociedad. Incluyendo cultura y comunicación.

- Noticias del corazón. Propias de la denominada prensa rosa.

- Ciencia y Tecnología.

- Medio Ambiente.

- Otros no especificados.

- Motivos que les induce a informarse. Planteados, entre otros, a partir de la necesidad o conexión con la profesión, de la idea de "estar al día", del interés por un determinado tema o por causas relacionadas con la vida social y familiar.

- Grado de interés y causas que les lleva a contrastar las noticias que reciben. La pregunta ¿Suele contrastar las noticias que recibe por primera vez a través de otro medio? fue recogida como variable tricotómica (sí, a veces, no) y acompañada, cada una de las alternativas, por tres posibles causas que les induce a mantener esta conducta. En este caso sirvió como respuesta filtro para conocer, si su respuesta no era negativa, a través de qué medio o medios contrastan las informaciones que reciben en primera instancia. 
- Frecuencia para distribuir noticias en su entorno. Igualmente esta cuestión sirvió como filtro de cara a conocer qué medio o medios utilizan para informar a las personas que integran su círculo social. Las alternativas que se expusieron para comprobar la frecuencia variaban desde "nunca" a "siempre". En segunda instancia y para conocer los medios que utilizan en este cometido, se plantearon las siguientes opciones: redes sociales, plataformas como What$s A p p$, cara a cara, por teléfono, blogs, otros.

- Percepción del grado de imparcialidad existente en los distintos medios de comunicación tradicionales, en sus versiones online y en medios sociales (blogs y redes sociales). Para este estudio, la imparcialidad se entiende como neutralidad o, en otras palabras, como ausencia de tendenciosidad en las informaciones difundidas.

- Grado de credibilidad que les inspiran los distintos medios de comunicación tradicionales, sus versiones online y los medios sociales (blogs y redes sociales). La credibilidad equivale a la confianza que transmite un determinado medio de comunicación en función de la veracidad que se aprecie en sus contenidos informativos.

Tanto en la percepción de imparcialidad como en el grado de credibilidad se utilizó la escala de Likert para determinar el valor que ofrecían de cada medio propuesto, tanto los online como los tradicionales. Para esta cuantificación, el 0 correspondía, respectivamente, a "ninguna credibilidad" y "nada imparcial", y el 5 a "total credibilidad" y "totalmente imparcial". El procesamiento de los datos se ha realizado con el programa estadístico SPSS en su versión 18.0.0. Los porcentajes y los totales que se exponen en el contenido de las tablas y de los gráficos se basan únicamente en las respuestas de los encuestados.

\section{Resultados}

\subsection{Preferencia para la consulta}

A pesar de que, en función de la edad de la muestra, todos los estudiantes están integrados en la generación de los nativos digitales, una amplia mayoría reconoce que sigue informándose a través de los medios tradicionales, siendo la televisión el medio referente en este apartado, seguida, por este orden, de la prensa escrita y de la radio. Solo un 9,1\% asegura que no consulta la información por alguna de estas vías.

Sin embargo, los jóvenes se decantan por el consumo de las formas digitales o generadas desde Internet, empleadas por el 99,6\% de los encuestados. Los medios sociales, Twitter y Facebook en mayor medida, constituyen el recurso de consulta más frecuente entre los universitarios, en un grado escasamente superior $(83,3 \%)$ entre los hombres que entre las mujeres $(82,4 \%)$; a un nivel notablemente más bajo se sitúa la prensa online $(69,6 \%)$ y el sistema de mensajería tipo WhatsApp $(31,1 \%)$, 
ambas vías son seguidas también más por el grupo masculino. El resto de los medios para la consulta son frecuentados por una franja que oscila entre el $25,4 \%$ de los buscadores -preferentemente Google- y el 1,8\% correspondiente a otras formas online no definidas.

Una línea similar se registra en función de la intensidad de la conexión a Internet, aunque las preferencias cambian entre los que se conectan menos de 1 hora diaria, los cuales optan por la prensa online en un $66,7 \%$ frente al $55,6 \%$ que señalan las redes sociales como vía de consulta; no obstante, en esta franja se registran únicamente 9 de los 286 casos válidos. En relación con esta variable, no se aprecia una sintonía entre una mayor navegación y la elección por un determinado medio online de consulta.

Tabla 1. Forma de consulta digital en función de la intensidad de navegación

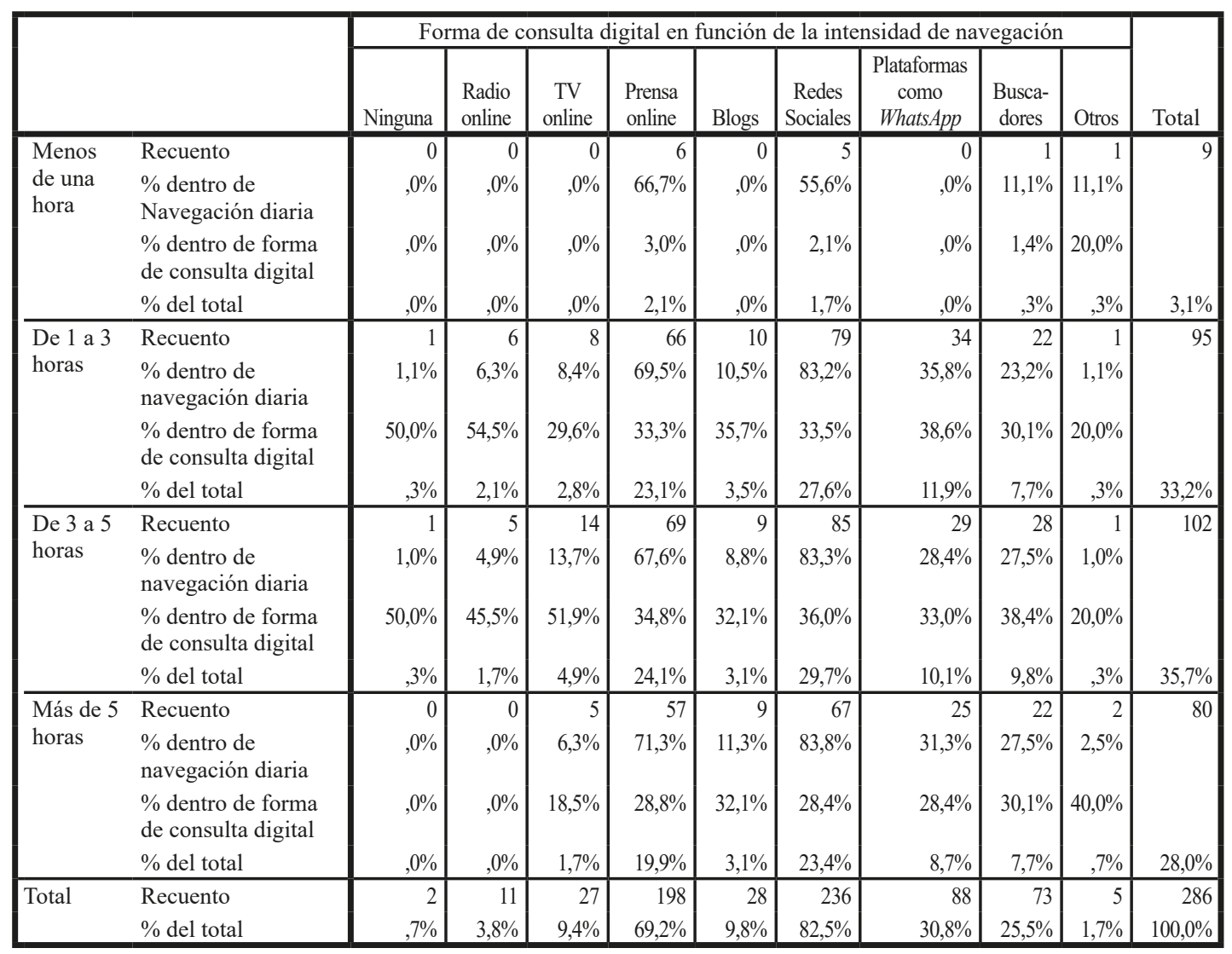

Fuente: Elaboración propia 


\subsection{Frecuencia de acceso}

La totalidad de los jóvenes encuestados navega habitualmente por Internet durante varias horas al día, tanto en jornadas laborables y/o lectivas como los fines de semana; en concreto, la moda de tiempo para ambas franjas semanales se sitúa en las 4 horas. Ateniéndonos a los porcentajes, se observa que, de lunes a viernes, más de un tercio de los jóvenes $(35,7 \%)$ está conectado de 3 a 5 horas y durante los fines de semana un $37,4 \%$ permanece navegando de 1 a 3 horas. Apenas es significativo que, en general, las cifras sobre el uso continuado de Internet bajan los sábados y domingos con respecto al resto de los días, cuestión que se demuestra con las respectivas medias registradas: 3,74 horas para la primera franja de tiempo considerada y 3,89 para la segunda.

Por otra parte, un $98 \%$ de los encuestados consulta habitualmente las noticias de actualidad aunque casi una cuarta parte $(24,7 \%)$ reconoce que no lo hace todos los días; del resto de la muestra que las consulta a diario, un 37\% lo hace una vez, 21,6\% dos veces y un $13,9 \%$ en tres o más ocasiones cada día. Estos porcentajes indican que la mayoría de los encuestados consulta la información una o dos veces al día, aunque en función del sexo se registran más mujeres que no hacen consultas diarias, $26,5 \%$ frente a un $19,4 \%$. Esta diferencia explica una inclinación mayor de hombres interesados por las noticias y que se refuerza con la tendencia de un $21,5 \%$ de este sector masculino que las consulta tres o más veces, porcentaje muy superior al 10,5 registrado entre las universitarias.

No se detecta una correspondencia entre la frecuencia en la consulta de noticias y el intervalo de tiempo de navegación por Internet. Los encuestados que están conectados entre 1 y 3 horas son los que muestran más interés por informarse $(37,6 \%)$ y, a su vez, son los que registran el porcentaje más alto $(46,9)$ del total que lo hacen dos veces al día. Si bien es cierto que los que navegan menos de una hora al día engloban al grupo más bajo de consulta $(6,8 \%)$ frente al $25,4 \%$ de los que permanecen frente a Internet más de cinco horas.

\subsection{Especialización de la información}

Sí se presentan diferencias por los distintos tipos de información en función del género. Los hombres muestran como primera opción las noticias de deportes mientras que las mujeres optan por la información sobre sociedad, cultura y comunicación. En esta línea, la mayor diferencia entre ambos grupos se sitúa también en sendas preferencias: un $36,8 \%$ en la deportiva y un $21 \%$ en la relacionada con sociedad, cultura y comunicación. La información política es consultada por un $58,2 \%$ de los hombres y por un $50 \%$ de las mujeres.

También resultan extremadamente significativas las fluctuaciones si se toma en consideración la intensidad en la navegación: los que usan Internet más de 5 horas se decantan en primer lugar por las noticias del corazón y, en segundo término, por las relacionadas con el medio ambiente. Aquellos que permanecen conectados entre $3 \mathrm{y}$ 
5 horas prefieren, por este orden, economía y política, mientras que los encuestados situados entre 1 y 3 horas eligen principalmente deportes y otras noticias de tipo no definido. En los casos de navegación inferior a una hora, las diferencias en los porcentajes registrados no son relevantes.

\subsection{Motivos para consultar las noticias}

La necesidad de cara a su profesión y/o a su futuro profesional es la causa principal que argumentan la práctica totalidad de los encuestados $(96,1 \%)$ para consultar las noticias; mientras que el mero hecho de estar al tanto de la información se sitúa en segundo lugar de sus motivaciones $(81,6 \%)$. A una importante distancia, les sigue, en este orden, el interés por una determinada temática $(36,8 \%)$ y para la toma de decisiones en diferentes ámbitos (30,2\%). Algo más de una quinta parte $(20,7 \%)$ adquirió el hábito en su entorno familiar y un $17,2 \%$ consulta las noticias para entablar conversaciones; finalmente hay un $2,7 \%$ que señala otras causas de naturaleza indeterminada.

No se aprecian diferencias importantes entre las razones esgrimidas por ambos sexos, salvo en el caso de su gusto por conocer información sobre un ámbito concreto: se decantan por ello un $49,4 \%$ de los hombres frente al $29 \%$ de las mujeres; en el resto de los motivos para la consulta las diferencias entre el grupo masculino y femenino oscilan en torno a los dos puntos porcentuales.

Tampoco se registran contrastes significativos en relación con la intensidad de acceso digital. Existe una sensible tendencia, no obstante, de los que navegan menos de 1 hora a decantarse por la opción de estar al día de las noticias $(90 \%)$ frente al $85 \%$ que argumenta la necesidad de cara a su profesión y/o futuro profesional. Por otro lado, el grupo conectado de 1 a 3 horas diarias opta algo más que el resto por la importancia que tiene la consulta para su toma de decisiones.

En el resto de las causas, la tendencia es similar a la línea de argumentos esgrimida por la totalidad de los encuestados; en consecuencia, no se observa que la intensidad en la navegación, igual que ocurre con el sexo, influye en la inclinación por uno u otro motivo para la consulta de noticias.

\subsection{Verificación de la información de actualidad}

La práctica totalidad de los hombres $(93,3 \%)$ y el $87,9 \%$ de las mujeres aseguran que ocasionalmente o siempre contrastan a través de otras vías las informaciones que consultan en primera instancia. No hay diferencias entre las cifras detectadas en uno y otro sexo que desarrollan esta práctica como un hábito; los índices sí bajan entre las mujeres frente a los hombres que únicamente contrastan a veces la información que consultan.

La confirmación de las versiones que les ofrece el primer medio se alza como la primera motivación de los hombres. Esto les lleva a contrastar las noticias habitualmente, mientras que las mujeres afirman que lo hacen preferentemente para completar con más datos. También existen diferencias causales entre los que contrastan la 
información esporádicamente: si bien el grupo masculino lo hace fundamentalmente cuando la información entraña cierta polémica, las mujeres desarrollan esta práctica si incide en su interés personal.

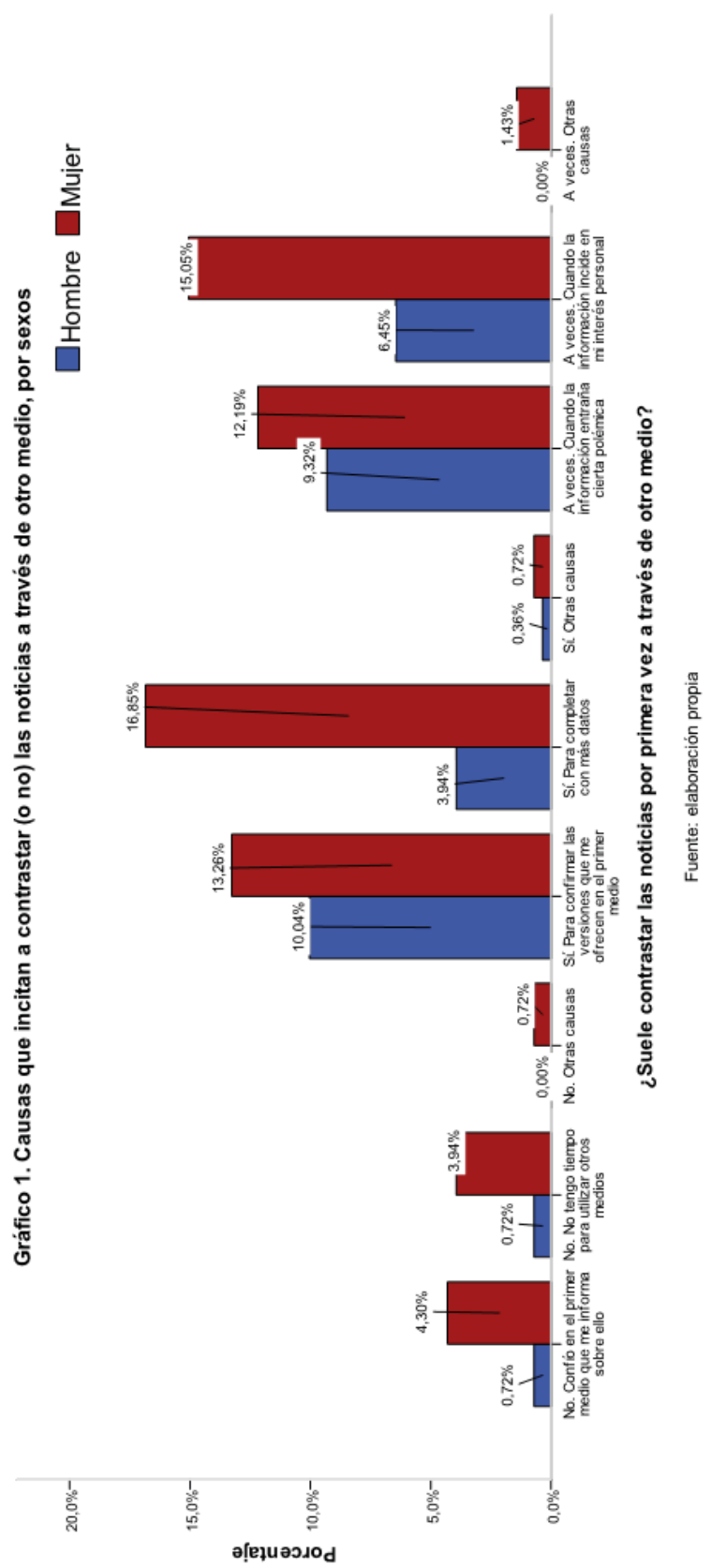


Los grupos más intensivos, los que se conectan a Internet de 3 a 5 horas y los que superan ese límite, se corresponden también con los que contrastan habitualmente a través de otros medios, aunque la proporción es mayor entre los primeros. Las cifras entre los que no desarrollan esta práctica oscila entre el $8,5 \%$ de los que navegan entre 1 a 3 horas y el 12,8\% de quienes lo hacen entre 3 a 5 horas. No existen diferencias relevantes de los grupos en lo que se refiere al contraste ocasional, aunque, en líneas generales, sí se observa que una superior intensidad en el uso de Internet marca una tendencia mayor a interesarse por la información generada por otras formas de difusión de noticias.

Resultan peculiares los porcentajes en la intensidad si observamos los motivos que les incitan a contrastar siempre las informaciones: los grupos extremos -menos de 1 hora y más de 5 horas de navegación- recurren en segunda instancia a otros medios para completar con más datos, mientras que los intermedios argumentan que su pretensión es confirmar las versiones que les ofrecen en la primera consulta a una información de actualidad.

\subsection{Distribución de noticias}

Los jóvenes universitarios no se conforman con ser meros receptores de la información: la práctica totalidad de los encuestados $(96,6 \%)$ reconoce que comparte con las personas de su entorno todas o algunas noticias a las que tienen acceso. La mayoría, un $42,2 \%$ lo hace casi siempre, el $33,1 \%$ en ocasiones y un 21,3 difunde siempre la información.

Sin embargo y al igual que ocurría con su tendencia a informarse a través de los medios tradicionales, los nativos digitales encuestados prefieren compartir las noticias con su entorno a través del cara a cara (un $84,7 \%$ se decanta por esta vía). Aunque plataformas como WhatsApp van tomando posición y se sitúan sensiblemente por encima de las redes sociales: $66,1 \%$ frente a $65,2 \%$. El teléfono y, finalmente, los blogs son utilizados de forma muy esporádica por la totalidad de los jóvenes.

Incluso aquellos que tienen una navegación intensiva de más de 5 horas diarias, prefieren estar presentes físicamente junto a las personas de su entorno, aunque en segundo lugar sí que optan por las redes sociales en detrimento de plataformas como Whatsapp. El resto de las clasificaciones de frecuencia de conexión online, siguen los parámetros generales mencionados anteriormente. En función del género, las mujeres son menos propensas a compartir la información y, a excepción del medio telefónico, utilizan en una proporción inferior el resto de las formas y vías para compartir información.

5.7. Percepción del grado de imparcialidad y credibilidad en los distintos medios

Algo más de un tercio de los encuestados califican de poco imparciales tanto los medios de comunicación tradicionales en sus diferentes formatos como los medios sociales en lo que se refiere al acceso a las noticias. Si bien la percepción de bastante imparcialidad es evidente para cerca de un $27 \%$ de los jóvenes, los valores superio- 
res de muy o totalmente imparciales son consignados únicamente por 1 de cada 10 encuestados.

Tabla 2. Percepción general de la imparcialidad en los medios tradicionales y online

\begin{tabular}{|c|c|c|c|c|}
\hline & Frecuencia & Porcentaje & $\begin{array}{l}\text { Porcentaje } \\
\text { válido }\end{array}$ & $\begin{array}{l}\text { Porcentaje } \\
\text { acumulado }\end{array}$ \\
\hline $\mathrm{NS} / \mathrm{NC}$ & 46 & 15,5 & 15,5 & 15,5 \\
\hline Nada imparcial & 26 & 8,8 & 8,8 & 24,2 \\
\hline Poco imparcial & 116 & 39,1 & 39,1 & 63,3 \\
\hline Bastante imparcial & 79 & 26,6 & 26,6 & 89,9 \\
\hline Muy imparcial & 19 & 6,4 & 6,4 & 96,3 \\
\hline Totalmente imparcial & 11 & 3,7 & 3,7 & 100,0 \\
\hline Total & 297 & 100,0 & 100,0 & \\
\hline
\end{tabular}

Fuente: elaboración propia

La valoración sobre la credibilidad que les inspiran los diferentes medios es más alta que la registrada en la tasas de imparcialidad. Prácticamente la mitad (un 40,7\%) considera a los medios bastante creíbles, aunque los porcentajes son muy cercanos si se tiene en cuenta la suma de los encuestados que les otorgan poca (32\%) o ninguna credibilidad (4\%). Las cifras descienden notablemente en el valor superior: menos de 1 de cada 10 encuestados considera al conjunto de los medios tradicionales y online como totalmente creíbles.

Tabla 3. Grado general de credibilidad en los medios tradicionales y online

\begin{tabular}{|l|r|r|r|r|}
\hline & Frecuencia & Porcentaje & \multicolumn{1}{c|}{$\begin{array}{c}\text { Porcentaje } \\
\text { válido }\end{array}$} & \multicolumn{2}{c|}{$\begin{array}{c}\text { Porcentaje } \\
\text { acumulado }\end{array}$} \\
\hline NS/NC & 42 & 14,1 & 14,1 & 14,1 \\
Ninguna credibilidad & 12 & 4,0 & 4,0 & 18,2 \\
Poca credibilidad & 95 & 32,0 & 32,0 & 50,2 \\
Bastante credibilidad & 121 & 40,7 & 40,7 & 90,9 \\
Mucha credibilidad & 23 & 7,7 & 7,7 & 98,7 \\
Total credibilidad & 4 & 1,3 & 1,3 & 100,0 \\
Total & 297 & 100,0 & 100,0 & \\
\hline
\end{tabular}

Fuente: elaboración propia

En una escala de Likert donde el valor 1 corresponde a nada imparcial y el 5 a totalmente imparcial, el medio peor considerado es la prensa escrita con 1,69 y el mejor la radio online con 2,69. Con la misma técnica y los mismos valores se observa que vuelve a ser este último medio el que inspira mayor credibilidad $(2,57)$, aunque el menos considerado lo constituyen las redes sociales que sólo alcanzan un valor medial de 1,57 . 
Gráfico 2. Medias de imparcialidad y credibilidad

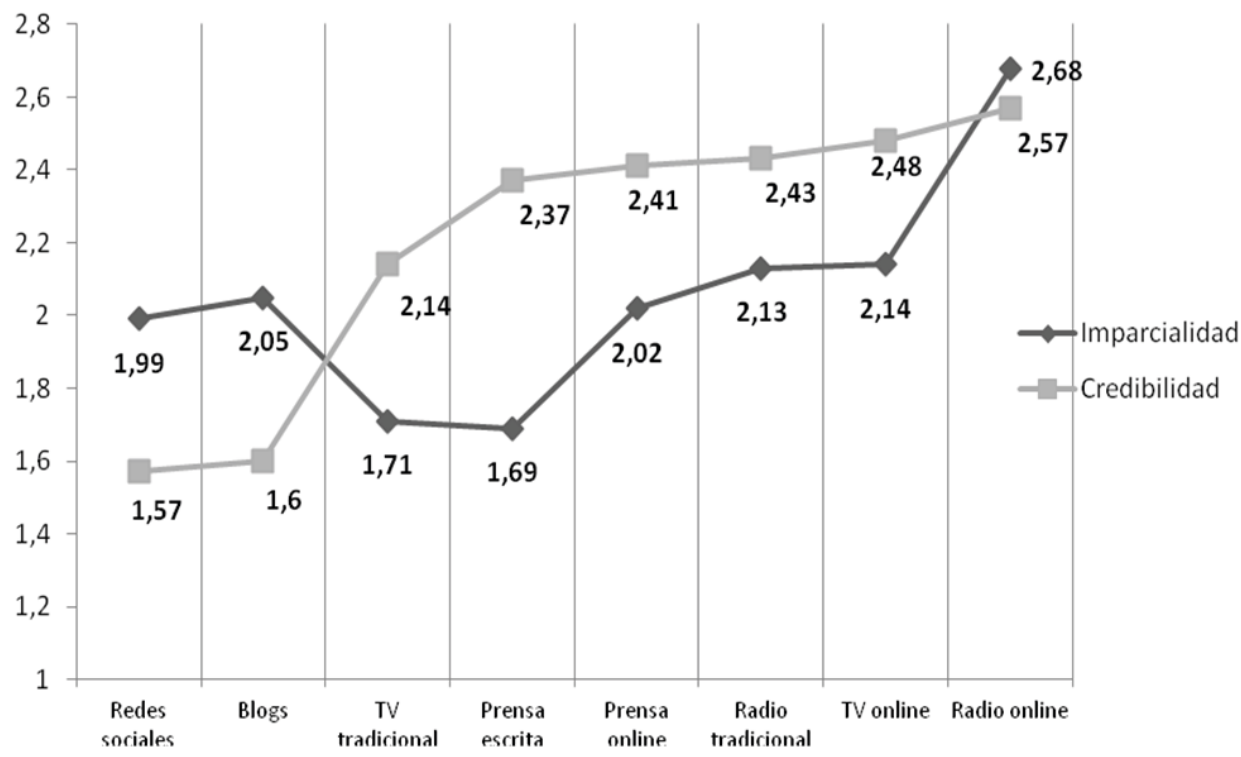

Fuente: elaboración propia

Por género, no hay diferencias significativas sobre la neutralidad en los medios tradicionales, a excepción de un mayor porcentaje de hombres que los consideran en mayor medida nada imparciales. En las versiones digitales de estos medios se observa una tendencia similar, aunque las mujeres consideran, más que ellos, que la televisión online no es imparcial. La misma apreciación tiene la población femenina de los blogs y de las redes sociales, en claro contraste con los hombres que dan una valoración mayoritaria de bastante o totalmente imparciales.

El grado de credibilidad de los medios tradicionales no varía entre ambos sectores de la población. Sí que registra una diferencia de 10 puntos por encima en la baja credibilidad que les inspira a las mujeres la radio y la televisión digitales, valoración que en parte se compensa con los 5 puntos de más que conceden los hombres a la prensa online. En cuanto a los medios sociales, Más de la mitad de las mujeres y algo más de un tercio de los hombres consideran que ni los blogs ni las redes aportan credibilidad alguna.

En relación con la intensidad de navegación tampoco se registran divergencias relevantes en el grado de imparcialidad que perciben de los medios, a excepción de las redes sociales que son consideradas totalmente imparciales por el $42,9 \%$ de los que navegan menos de 1 hora frente al $12,6 \%$ de media que señalan con esta misma calificación el resto de las franjas establecidas. 
Finalmente, solo se aprecia un rasgo diferenciador significativo en la credibilidad para la radio online: un tercio de los encuestados que navega menos de 1 hora al día considera que este medio no tiene ninguna credibilidad frente a la misma valoración que señalan el 15,2\% de los que están conectados diariamente a Internet durante más tiempo. En el resto de los medios no se aprecia significaciones y, en consecuencia, por lo general, la intensidad de navegación no influye en las valoraciones de credibilidad que inspiran todos los medios.

\section{Conclusiones y discusión}

A tenor del análisis de los resultados registrados y conociendo las limitaciones muestrales, se pueden extraer las siguientes conclusiones:

Primera. Los jóvenes universitarios encuestados, que se encuentran dentro del espectro de los denominados nativos digitales, recurren preferentemente a Internet para informarse, aunque se mantiene una marcada inclinación de la práctica totalidad por alternar esta consulta de noticias con los medios tradicionales. Al margen de otros usos que realicen de las redes sociales, se observa una importante tendencia a consultar las noticias distribuidas (o difundidas) desde Facebook y Twitter, pero no se corrobora la primera hipótesis que enunciaba la preferencia por el consumo de los medios sociales como elemento informativo. Los resultados del estudio confirman la tendencia ascendente de Twitter, enunciada por Kim et al. (2014), que auguraban un incremento del uso de este medio como espacio de localización de información.

Los medios convencionales, en especial la televisión, se mantienen como una importante opción referencial. De este modo, los medios sociales, junto con la televisión, se configuran como las herramientas preferidas por los jóvenes universitarios para informarse; unos resultados en sintonía con los obtenidos por Condeza, Bachmann y Mujica (2014) en su estudio sobre los adolescentes, siendo la principal diferencia el mayor uso de Twitter entre los jóvenes.

Segunda. El consumo de noticias queda condicionado por la intensidad del acceso digital. Por lo que respecta al intervalo de tiempo diario que los usuarios se conectan a Internet, queda demostrado que si el acceso es inferior a una hora, el consumo de información no es una prioridad, siendo el intervalo entre 1 y 3 horas el de mayor consumo (coincidente con una preferencia temática por la información deportiva), puesto que en mayores rangos no se aprecia un aumento proporcional de acceso a las noticias, lo que indica la preferencia por otros usos de Internet. Entre tres y cinco horas las temáticas más consultadas son de economía y política, mientras que cuando se superan las 5 horas interesan los temas del corazón y medio ambiente.

En lo que respecta a la motivación de la consulta, la diversidad de intereses no parece asociarse al tiempo de acceso a Internet, si bien los usuarios que se sitúan en el intervalo de 1 a 3 horas son los que esgrimen como motivación, en mayor porcentaje 
que el resto, que su consulta de la información está condicionada a mejorar el proceso de toma de decisiones.

Por otra parte, puesto que contrastar las informaciones es una tarea que requiere su tiempo, los resultados que relacionan la mayor intensidad en el uso de Internet con un mayor contraste de la información constatan un lógico paralelismo. Sorprende, sin embargo, que con independencia del tiempo que pase un usuario navegando (ya sea menos de una hora o más de cinco) hay una evidente preferencia por compartir la información de manera presencial, cara a cara, y esto a pesar de realizar un estudio entre los nativos digitales.

Finalmente, la intensidad de la navegación sólo tiene una significativa influencia en las variables de imparcialidad y credibilidad entre los usuarios que se conectan a Internet menos de una hora. Estos jóvenes atribuyen un alto grado de imparcialidad a las redes sociales, así como ninguna credibilidad a la radio online, unas consideraciones exclusivas para los jóvenes integrados en este intervalo de conexión, pues en el resto de casos las percepciones sobre imparcialidad y credibilidad de los diferentes medios resulta homogénea.

Tercera. Las diferencias a partir del género no son significativas en la mayor parte de las variables analizadas, tal y como propone la tercera hipótesis, con excepción de la temática de consulta entre las que destaca la preferencia de los varones por la información deportiva, mientras que las mujeres se decantan por las noticias sobre sociedad, cultura y comunicación. Sin embargo, la práctica totalidad de los encuestados afirma que la principal motivación para consultar la información es el necesario conocimiento de la actualidad que requiere su ámbito profesional, de lo que se deduce que más de un tercio de los hombres estaría interesado en buscar una salida profesional asociada con el ámbito deportivo.

Otra diferencia de género que se podría destacar sería la motivación que lleva a los jóvenes a contrastar las fuentes informativas, pues en el caso de los hombres lo que se persigue es corroborar la primera información consultada, mientras que en el caso de las mujeres la principal motivación para una segunda consulta es la de completar los datos.

En el resto de variables y subvariables, se puede afirmar que no existen diferencias significativas en cuestiones de género de modo que, en el tipo de usuario analizado, no se aprecian brechas de género como las extraídas por Casero Ripollés (2012) en su investigación.

Las conclusiones de esta investigación tienen un carácter exploratorio y limitado. Es pertinente ampliar el universo y acometer una investigación con una muestra probabilística que recoja el comportamiento digital de todos los jóvenes, equilibrando los números absolutos de participación de hombres y mujeres. Una de las cuestiones que se puede aprovechar en el análisis de aquellos jóvenes que se manifiestan poco o nada propensos al consumo de noticias, con el fin de obtener algunos datos sobre el porqué no se despierta en ellos ningún interés por la información de actualidad. 


\section{Referencias bibliográficas}

CASERO-RIPOLLÉS, A. (2012). Más allá de los diarios: el consumo de noticias de los jóvenes en la era digital. Comunicar, 39(XX), pp. 151-158. doi: 10.3916/ C39-2012-03-05

CONDEZA, R.; BACHMANN, I.; MUJICA, C. (2014). El consumo de noticias de los adolescentes chilenos: intereses, motivaciones y percepciones sobre la agenda informativa. Comunicar, 43(XXII), pp. 55-64. doi: http://dx.doi.org/10.3916/ C43-2014-05

COSTERA, I. (2007). The Paradox of Popularity: How Young People Experience the News. Journalism Studies, 8(1), pp 96-116. doi: 10.1080/14616700601056874.

FOGG ,B.J. (2002). Promince-Interpretation Theory: Explaining How People Assess Credibility Online. CHI2003 Extended Abstracts. New York: ACM Press. En línea: http://credibility.stanford.edu/pdf/p-iTheory_Fogg_Oct02.pdf.

GANGADHARBATLA, H.; BRIGHT, L. F.; LOGAN, K. (2014). Social Media and news gathering: tapping into the millennial mindset. The Journal of Social Media in Society, 3(1), pp. 45-63.

GARCÍA AVILÉS, J. A.; NAVARRO MAILLO, F.; ARIAS ROBLES, F. (2014). La credibilidad de los contenidos informativos en internet para los 'nativos digitales': estudio de caso. Palabra Clave, 17(3), pp. 875-894. doi: 10.5294/ pacla.2014.17.3.13

GARCÍA GARCÍA, F., GÉRTRUDRIX BARRIO, M.; GÉRTRUDRIX BARRIO, F. (2014). Análisis de la incidencia de la dieta de servicios digitales en la utilidad y confianza de la información en internet en los jóvenes universitarios. Communication \& Society / Comunicación y Sociedad, 27(1), pp. 59-81.

GIL DE ZÚÑIGA, H.; JUNG, N.; VALENZUELA, S. (2012). Social media use for news and individuas's social capital, civic engagement and political participation. Journal of Computer-Mediated Communication, 17, pp. 319-336. doi:10.1111/ j.1083-6101.2012.01574.x

HASEBRINK, U.; DOMEYER, H. (2012). Media repertoires as patterns of behaviour and as meaningful practices: A multimethod approach to media use in converging media environments. Participations. Journal of Audience \& Reception Studies, 9(2), pp. 757-779.

HERMIDA, A.; FLETCHER, F.; KORELL, D.; LOGAN, D. (2012). Share, like, recommend. Decoding the social media news consumer. Journalism Studies, 13(5-6), pp. 815-824. doi: 10.1080/1461670X.2012.664430

JOHNSON, T. J.; KAYE B. K. (2014). Credibility of social network sites for political information among politically interested internet users. Journal of Computer-mediated communication, 19, pp. 957-974. doi: 10.1111/jcc4.12084

KIM, K.-S., SIN, S.-C. J.; HE, Y. (2013). Information seeking through social media: impact of user characteristics on social media use. Proceedings of the American Society for Information Science and Technology, 50(1), pp. 1-4.

KIM, K.-S., SIN, S.-C. J.; YOO-LEE, E. (2014). Undergraduates' use of social media as information sources. College \& Research Libraries, 75(4), pp. 442-457. 
MELTON, J., MILLER, R.; SALMONA, M. (2012). Online social networks: Student perceptions and behavior across four countries. International Journal of Information Systems \& Social Change, 3(2), pp. 24-38. doi: 10.4018/ijissc.2012040102

PATTERSON, T.E. (2007). Young People and News. Joan Sho renstein Center on the Press, Politics and Public Policy. Harvard University, John F. Kennedy School of Government. En línea: http://shorensteincenter.org/wp-content/uploads/2012/03/ young_people_and_news_2007.pdf

SÁNCHEZ-NAVARR̄O, J.; ARANDA, D. (2011). Internet como fuente de información para la vida cotidiana de los jóvenes españoles. El profesional de la información, 20(1), pp. 32-37. doi: 10.3145/epi.2011.ene.04

TÚÑEZ, M. (2009). Jóvenes y prensa en papel en la era de Internet. Estudio de hábitos de lectura, criterios de jerarquía de noticias, satisfacción con los contenidos informativos y ausencias temáticas. Estudios sobre el Mensaje Periodístico, 15, pp. 503-524.

TURCOTTE, J.; YORK, C.; IRVING, J.; SCHOLL, R. M.; PINGRE, R.J. (2015). News recommendations from social media opinion leaders: effects on media trust and information seeking. Journal of Computer-Mediated Communication. doi: $10.1111 /$ jec 4.12127 